\title{
Article
}

\section{Sulforaphane Mediates Glutathione Depletion via Polymeric Nanoparticles to Restore Cisplatin Chemosensitivity}

Ying Xu, Xuexiang Han, Yiye Li, Huan Min, Xiao Zhao, Yinlong Zhang, Yingqiu Qi, Jian Shi, Sheng Qi, Yongping Bao, and Guangjun Nie

ACS Nano, Just Accepted Manuscript • DOI: 10.1021/acsnano.9b07032 • Publication Date (Web): 31 Oct 2019

Downloaded from pubs.acs.org on November 1, 2019

\section{Just Accepted}

"Just Accepted" manuscripts have been peer-reviewed and accepted for publication. They are posted online prior to technical editing, formatting for publication and author proofing. The American Chemical Society provides "Just Accepted" as a service to the research community to expedite the dissemination of scientific material as soon as possible after acceptance. "Just Accepted" manuscripts appear in full in PDF format accompanied by an HTML abstract. "Just Accepted" manuscripts have been fully peer reviewed, but should not be considered the official version of record. They are citable by the Digital Object Identifier (DOI®). "Just Accepted" is an optional service offered to authors. Therefore, the "Just Accepted" Web site may not include all articles that will be published in the journal. After a manuscript is technically edited and formatted, it will be removed from the "Just Accepted" Web site and published as an ASAP article. Note that technical editing may introduce minor changes to the manuscript text and/or graphics which could affect content, and all legal disclaimers and ethical guidelines that apply to the journal pertain. ACS cannot be held responsible for errors or consequences arising from the use of information contained in these "Just Accepted" manuscripts. 


\section{Sulforaphane Mediates Glutathione Depletion via Polymeric Nanoparticles to Restore Cisplatin Chemosensitivity}

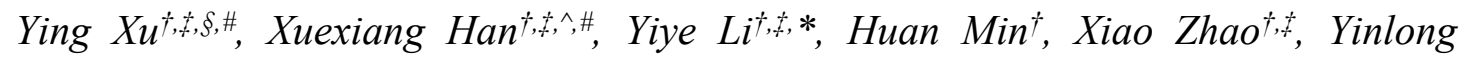
Zhang ${ }^{\dagger, t}$, Yingqiu ${ }^{\perp} i^{\perp}$, Jian Shit, Sheng ${ }^{\dagger}{ }^{\otimes}$, Yongping Bao ${ }^{\nabla, *}$, Guangjun Nie ${ }^{\dagger, t, *}$

CAS Key Laboratory for Biomedical Effects of Nanomaterials and Nanosafety, CAS Center for Excellence in Nanoscience, National Center for Nanoscience and Technology, Beijing 100190, P.R. China

\#enter of Materials Science and Optoelectronics Engineering, University of Chinese Academy of Sciences, Beijing 100049, P.R. China

${ }^{\S}$ Sino-Danish Center for Education and Research, Sino-Danish College of University of Chinese Academy of Sciences, Beijing 100049, P.R. China

${ }^{\wedge}$ Department of Chemistry, Tsinghua University, Beijing 100084, P.R. China

$\perp$ School of Basic Medical Sciences, Zhengzhou University, Zhengzhou Henan 450001, PR. China

${ }^{\otimes}$ School of Pharmacy, University of East Anglia, Norwich, Norfolk, NR4 7TJ, UK

${ }^{\nabla}$ Norwich Medical School, University of East Anglia, Norwich, Norfolk, NR4 7UQ UK

\#These authors contributed equally to this work.

*To whom correspondence may be addressed: liyy@nanoctr.cn (Yiye Li), y.bao@uea.ac.uk (Yongping Bao),niegj@nanoctr.cn (Guangjun Nie). 


\begin{abstract}
Platinum (Pt)-based chemotherapy is a broadly used therapeutic regimen against various cancers. However, the insufficient cellular uptake, deactivation by thiol-containing species and nonspecific distribution of cisplatin (CDDP) result in its low chemosensitivity as well as systemic side effects, which can largely constrain the employment of CDDP in clinical treatment. To circumvent these problems, in this study, polymeric nanoparticles were utilized to co-deliver a water-soluble CDDP derivative, poly ( $\gamma$, L-glutamic acid)-CDDP conjugate, and a naturally occurring compound derived from broccoli, sulforaphane, which can achieve efficient glutathione (GSH) depletion, to improve the accumulation of CDDP in cancer cells. Results show that compared with combinational treatment of CDDP and SFN, the nanoparticles were more effectively internalized and could significantly reduce GSH content in breast cancer cells, leading to a notable increase in DNA-bound Pt and DNA damage-induced apoptosis. Moreover, in an orthotopic breast cancer model, the nanoparticles achieved a significantly higher tumor accumulation and exhibited a more powerful anti-tumor activity. Finally, this nano-enhanced chemotherapy was further confirmed in a liver cancer model with high-expression of GSH. Taken together, this sulforaphane-based nano-strategy holds great promise to enhance the sensitivity and therapeutic efficacy of Pt-based chemotherapy.
\end{abstract}

KEYWORDS: breast cancer, sulforaphane, cisplatin, nanoparticle, glutathione 
Breast cancer is one of the most common malignancies and the leading cause of cancer-related mortality in women. ${ }^{1}$ The platinum $(\mathrm{Pt})$ anti-cancer agent cisplatin (cis-dichlorodiammineplatinum (II), CDDP) is broadly used alone or in combination with other therapeutics to combat breast cancer and other human cancers in clinic. ${ }^{2}$ CDDP functions through forming both inter- and intra-strand crosslinks of nuclear DNA, which leads to DNA damage and cell apoptosis. ${ }^{3-5}$ Unfortunately, CDDP indiscriminately damages both tumor cells and normal cells following systemic administration, ${ }^{6}$ and exhibits dose-limiting side effects such as acute nephrotoxicity and hepatotoxicity, ${ }^{4,7}$ which largely restricts its clinical use.

Over the last two decades, nanoparticles (NPs)-enabled drug delivery systems have greatly advanced Pt-based chemotherapy. ${ }^{8,9}$ This delivery strategy is based on observations that NPs with a size between 20-200 nm can prolong the blood circulation of the loaded therapeutic agents and preferentially enhance tumor accumulation with reduced nonspecific distribution in healthy tissues owning to the enhanced permeability and retention (EPR) effect. ${ }^{10-12} \mathrm{NPs}$, such as long-circulating liposomes, ${ }^{13,14}$ water-soluble polymers, ${ }^{15,16}$ and polymeric micelles, ${ }^{17-19}$ have been widely utilized to facilitate CDDP delivery to the site of action. To date, poly (L-glutamic acid) is the most successful CDDP carrier. ${ }^{19}$ Particularly, the poly(ethylene glycol)-poly(L-glutamic acid) block copolymers, which can form polymeric micelles (NC-6004, developed by Nanocarriers ${ }^{\circledR}$ ) after incorporating CDDP through the polymer-metal complex formation between carboxyl groups and CDDP, have entered phase III clinical trials in Japan and other Asian countries. ${ }^{19,20}$ 
Nevertheless, despite that NC-6004 and other nano-formulations have been demonstrated to markedly reduce the systemic toxicity of CDDP, the therapeutic efficacy has not significantly improved..$^{7,21}$

One of the most important reasons for the unsatisfactory anti-tumor effect of Pt-based chemotherapy is the cytoplasmic detoxification of CDDP by elevated levels of thiol-containing species. $^{22,23}$ Glutathione (GSH), the most abundant thiol-containing molecule in cells, can avidly bind with $\mathrm{Pt}$ to form $\mathrm{Pt}(\mathrm{GS})_{2}$ conjugates, ${ }^{5}$ which are rapidly exported from tumor cells via ATP-dependent glutathione S-conjugate export pumps (GS-X pump).${ }^{24}$ Numerous studies have proven that the chemosensitivity of cancer cells to CDDP can be largely restored by reducing the cellular concentration of GSH. ${ }^{4,19}$ For example, Chen and colleagues demonstrated that disulfiram, a prescription drug used to treat alcoholism, could decrease intracellular GSH and enhance CDDP efficacy. ${ }^{19}$ Sulforaphane (SFN), a naturally occurring isothiocyanate enriched in broccoli, is well-known for its cancer prevention effect, of which the mechanisms involve multiple targeted effects, including the induction of phase II antioxidant enzymes, cell cycle arrest and cellular apoptosis. ${ }^{25-29}$ Moreover, SFN has been reported to deplete GSH by directly binding with GSH to form GSH-SFN complex, which can be further exported outside the cell. ${ }^{30,31}$ Therefore, SFN has great translational potential for sensitizing Pt-based chemotherapy in a safe manner. Considering the aforementioned negative correlation between GSH content and tumor chemosensitivity, we propose that the therapeutic efficacy of 
nano-formulated CDDP can be significantly improved by SFN-mediated GSH depletion, a hypothesis has not been explored so far.

Here, a NPs-enabled co-delivery system consisting of a water-soluble $\operatorname{poly}(\gamma$, L-glutamic acid)-CDDP ( $\gamma$-PGA-CDDP) conjugate and SFN for breast cancer treatment is reported. NPs encapsulating two drugs (termed as SFN-CDDP-NPs) are prepared with amphiphilic copolymer methoxy poly(ethylene glycol)-poly(lactide-co-glycolide) (mPEG-PLGA), which can simultaneously deliver hydrophobic and hydrophilic drugs to the same cancer cell with a predetermined ratio after a single injection. ${ }^{32}$ Because of the better solubility and much higher encapsulation efficiency relative to CDDP, ${ }^{15,33}$ the $\gamma$-PGA-CDDP, formed by coordination interaction between carboxyl groups of $\gamma$-PGA and CDDP, is encapsulated into the hydrophilic core. Meanwhile, the hydrophobic SFN is loaded into the hydrophobic shell. The therapeutic efficacy of SFN-CDDP-NPs is systematically investigated and compared with free drugs and their combination both in vitro and in vivo. Moreover, mechanism studies, including quantification of cellular GSH contents and DNA-bound Pt, analysis of DNA damage and apoptosis related proteins are performed to better understand the role of SFN in sensitizing CDDP.

\section{RESULTS AND DISCUSSION}

Synthesis and Characterization of SFN-CDDP-NPs. The water-soluble $\gamma$-PGA-CDDP conjugate was first prepared according to published methods, ${ }^{34}$ and successful synthesis was confirmed by Fourier transformed infrared (FT-IR) results 
(Figure S1). SFN and $\gamma$-PGA-CDDP loaded mPEG-PLGA nanoparticles (SFN-CDDP-NPs) were fabricated using an adapted double emulsion method, ${ }^{32,34}$ with hydrophilic $\gamma$-PGA-CDDP trapped in the hydrophilic core and hydrophobic SFN inserted in the hydrophobic layer (Scheme 1). The encapsulation efficiencies of SFN and CDDP in the optimized SFN-CDDP-NPs were $58.7 \pm 4.5 \%$ and $92.6 \pm 3.6 \%$, respectively (Table S1), which gave an approximate molar ratio of 1:2 for SFN/CDDP. Transmission electron microscopy (TEM) images revealed a uniform spherical morphology of the NPs with the diameter of $\sim 75.4 \mathrm{~nm}$ for blank mPEG-PLGA NPs and $\sim 82.3 \mathrm{~nm}$ for CDDP-SFN-NPs (Figure S2a and 1a). The dynamic light scattering (DLS) results revealed that the hydrodynamic size of mPEG-PLGA NPs was $~ 93.4$ $\mathrm{nm}$ with ca. $-14.4 \mathrm{mV}$ of the zeta potential (Figure S2b,c). However, SFN-CDDP-NPs exhibited a slightly larger hydrodynamic diameter of $\sim 98.2 \mathrm{~nm}$ with ca. $-15.4 \mathrm{mV}$ of the zeta potential (Figure 1b,c), which are supposed to assist in long blood circulation and passive tumor targeting. ${ }^{36,37}$ Furthermore, SFN-CDDP-NPs showed a good colloidal stability and maintained the structural integrity after incubation at $37{ }^{\circ} \mathrm{C}$ for 3 days (Figure S3). Next, the drug release profile of SFN-CDDP-NPs was studied at pH 5.5 or 7.4 (Figure 1d). Both SFN and CDDP showed sustained release profiles within $48 \mathrm{~h}$ and exhibited higher release rates at $\mathrm{pH} 5.5$ than 7.4. Notably, SFN was released from SFN-CDDP-NPs more rapidly than CDDP, which was favorable for decreasing intracellular GSH and preventing CDDP deactivation.

Cellular Uptake and Intracellular Localization of NPs. In order to reveal the cellular uptake behavior of NPs, rhodamin B-labeled mPEG-PLGA NPs (RhoB-NPs) 
with similar physicochemical properties to SFN-CDDP-NPs were prepared (Figure S4). Free RhoB and RhoB-NPs were incubated with MCF-7 cells and then observed using a confocal laser scanning microscope. As illustrated in Figure 2a, both free RhoB and RhoB-NPs showed time-dependent cellular uptake. At $1 \mathrm{~h}$ post-treatment, most of red fluorescence from RhoB-NPs was co-located with green fluorescence (LysoTraker Green stained lysosomes), indicating that RhoB-NPs were mainly internalized through endocytosis and entered into lysosomes. After a continuous internalization of RhoB-NPs for $6 \mathrm{~h}$, abundant red fluorescence separated with the green fluorescence, suggesting their translocation from lysosomes to cytoplasm. By contrast, free RhoB was uniformly distributed in the cytoplasm all the time, indicating that small molecules were passively diffused into cells. In addition, the cells treated with RhoB-NPs showed a stronger RhoB signal in comparison with free RhoB group, most likely due to the more efficient endocytosis of NPs than passive diffusion of small molecules. ${ }^{38}$ The enhanced internalization of NPs was further confirmed by quantitative analysis using flow cytometry, where significantly higher RhoB signal was detected in RhoB-NPs treated cells compared to free RhoB treated cells (Figure $2 b, c)$.

Enhanced Cytotoxicity in Vitro. The cytotoxicity of different formulations was studied using a cell counting kit-8 (CCK-8) assay. Consistent with the known biocompatibility of amphiphilic copolymer mPEG-PLGA, ${ }^{39}$ the mPEG-PLGA NPs exhibited no noticeable cytotoxicity in MCF-7 cells even at concentrations up to 800 $\mu \mathrm{g} / \mathrm{mL}$ (Figure S5), which was much higher than the dose used in the study. Although 
CDDP exhibited dose-dependent toxicity towards MCF-7 cells (Figure 3a), the efficacy of CDDP was relative low with an IC50 of $72.59 \mu \mathrm{M}$ (Table S2). SFN largely improved the chemosensitivity of CDDP with an IC50 of $48.97 \mu \mathrm{M}$ for SFN+CDDP group. Encouragingly, SFN-CDDP-NPs achieved the most significant cytotoxicity with an IC50 as low as $11.59 \mu \mathrm{M}$, which is contributed to its enhanced cellular uptake compared with the free drug. Cell apoptosis is identified as the basic pharmacodynamic effect of CDDP. ${ }^{5}$ The apoptosis triggered by SFN-CDDP-NPs was determined quantitatively by flow cytometry analysis after Annexin V-FITC/PI co-staining (Figure 3b,c). The results showed that CDDP induced moderate apoptosis of MCF-7 cells with an apoptotic rate of $15.2 \%$. By contrast, combination of SFN and CDDP raised the apoptotic rate to $39.5 \%$. The most noteworthy result was SFN-CDDP-NPs, with a maximal apoptotic rate of $64.6 \%$.

Large amounts of studies have showed that CDDP-mediated DNA damage could activate p53 and further trigger p53-mediated apoptosis as well as PARP cleavage-mediated cellular decomposition. ${ }^{4,40}$ To confirm the mechanism of our nano-strategy, western blot analysis was conducted to analyze the levels of apoptosis related proteins. As shown in Figure 4a,b, the expression of $\gamma$-H2AX (marker of DNA damage), p53 and cleaved PARP were moderately up-regulated after CDDP treatment, suggesting a moderate increase of cell apoptosis. However, SFN+CDDP treatment led to a more significant increased expression of these apoptosis related proteins, demonstrating the supportive role of SFN to augment CDDP-mediated cell apoptosis. Unsurprisingly, the advantage of this combined formulation was further amplified by 
NPs-enabled drug co-delivery, as SFN-CDDP-NPs treatment resulted in the most profound up-regulation of $\gamma$-H2AX, p53 and cleaved PARP. It is to be noted, anti-apoptotic protein Bcl-2 was slightly increased after CDDP treatment, which could be ascribed to the rescue response of cells and was correlated with CDDP tolerance. ${ }^{22}$ However, SFN treatment significantly inhibited CDDP-mediated Bcl-2 up-regulation, which was in accordance with previous reports. ${ }^{41,42}$ Encouragingly, SFN-CDDP-NPs treatment led to the most notable reduction of Bcl-2 expression. These results strongly confirm the cell apoptosis mechanism of the significant cytotoxicity of SFN-CDDP-NPs.

Increased Pt-DNA Complex by GSH depletion. GSH plays a vital role in CDDP detoxification, and depletion of GSH is suggested to increase the formation of Pt-DNA adducts and the chemosensitivity. ${ }^{4,19}$ To study the efficacy of SFN-mediated GSH depletion, the cellular GSH and DNA-bound Pt were measured after different treatments. The results showed that SFN effectively reduced GSH content in MCF-7 cells (Figure 4c). Compared to free SFN and SFN+CDDP, SFN-CDDP-NPs achieved more profound depletion of GSH, presumably due to more efficient cellular uptake of NPs than free drug (Figure 2). The quantification of Pt content in the genomic DNA of MCF-7 cells revealed that, with the help of SFN-mediated GSH depletion, SFN+CDDP enabled more accumulation of DNA-bound Pt than CDDP (Figure 4d). Encouragingly, SFN-CDDP-NPs treatment resulted in the highest level of DNA-bound Pt, which led to the most significant cell cytotoxicity (Figure 4e). However, pretreatment with N-acetylcysteine (NAC, a precursor of GSH) largely 
reduced the cytotoxicity of CDDP, SFN+CDDP and SFN-CDDP-NPs, which further emphasized the significance of GSH depletion to CDDP sensitization. Together, these results strongly suggest that robust depletion of GSH is indispensable for the increased formation of Pt-DNA adducts, which accounts for the superior therapeutic efficacy of SFN-CDDP-NPs in vitro.

Pharmacokinetics and Bio-Distribution in Vivo. The enhanced cytotoxicity in vitro prompted the evaluation of the therapeutic potential of SFN-CDDP-NPs in vivo. In order to reveal the pharmacokinetics and bio-distribution of NPs, indocyanine green-labeled mPEG-PLGA NPs (ICG-NPs) with similar physicochemical properties to SFN-CDDP-NPs were prepared (Figure S6). Free ICG and ICG-NPs were intravenously administrated into tumor-bearing mice and blood was withdrawn at predetermined time points. As shown in Figure S7, free ICG was rapidly eliminated from the blood. However, ICG-NPs were cleared much more slowly due to the prolonged blood circulation. ${ }^{34}$ In vivo whole-body imaging of tumor-bearing mice also confirmed the slower elimination and longer tumor retention of ICG-NPs than free ICG (Figure 5a). Ex vivo imaging results illustrated a much stronger fluorescent intensity of ICG-NPs in tumors compared to free ICG treatment (Figure 5b), which was benefited from the prolonged blood circulation and from the EPR effect-mediated tumor accumulation. ${ }^{11,43}$ The extended circulation time and greater tumor accumulation of SFN-CDDP-NPs compared to CDDP were further confirmed by quantification of $\mathrm{Pt}$ content in the blood, major organs and tumors using ICP-MS 
(Figure S8). Taken together, SFN-CDDP-NPs can maintain higher effective drug concentrations and sustain a longer therapeutic period than free drugs.

Anti-Tumor Therapy. The therapeutic effect of SFN-CDDP-NPs was investigated in an orthotopic breast tumor model. MCF-7 tumor-bearing mice were administrated with saline, SFN, CDDP, SFN+CDDP, SFN-CDDP-NPs, or SFN-CDDP-NPs/NAC. The tumor size increased rapidly in the saline group (Figure 5c), but CDDP treatment only moderately inhibited tumor progress with a tumor inhibition rate (TIR) of $39.1 \%$ (Figure 5d,e,f). Unlike the in vitro results, SFN barely enhanced the anti-tumor effect of CDDP (TIR $=47.2 \%$ ) most likely due to the rapid elimination of small molecules (Figure S7). On the contrary, the tumor growth was more significantly inhibited in the SFN-CDDP-NPs group with a greater TIR of $74.1 \%$. Additionally, pretreatment with NAC almost abolished the therapeutic benefit of SFN-CDDP-NPs, further emphasizing the key role of SFN for increased CDDP chemosensitivity. Moreover, the quantification of Pt content in tumors confirmed the highest CDDP accumulation in tumors of SFN-CDDP-NPs group (Figure S9a). Specifically, the quantification of Pt content in the genomic DNA of tumors revealed the greatest amount of DNA-bound Pt in SFN-CDDP-NPs group (Figure S9b). Therefore, the superior anti-tumor effect of SFN-CDDP-NPs is mainly ascribed to the significant increase of CDDP accumulation and DNA platination via NPs-mediated passive targeting and SFN-based GSH depletion. 
To reveal the molecular mechanism of enhanced anti-tumor efficacy provided by SFN-CDDP-NPs, tumor sections were subjected to histopathological examination (Figure 6a,b). Hematoxylin and eosin (H\&E) staining results showed that SFN-CDDP-NPs treatment caused the most cell death with a looser cell arrangement. The most significant increase in apoptosis related proteins $\gamma$-H2AX, p53 and cleaved PARP, as revealed by immunohistochemical (IHC) staining, was observed in tumor tissues after SFN-CDDP-NPs treatment. In addition, the up-regulation of anti-apoptotic protein Bcl-2 in response to CDDP treatment was significantly inhibited in the SFN-CDDP-NPs group. As a result, the most notable level of apoptosis was observed in the SFN-CDDP-NPs group with a highest percentage of TUNEL-positive cells (Figure 6c,d).

Afterwards, the safety profile of SFN-CDDP-NPs was evaluated. The mice treated with CDDP or SFN+CDDP exhibited a slight body weight loss after three intravenous injections (Figure S10), which is a commonly reported side effect of CDDP. ${ }^{4}$ However, this was not observed in SFN-CDDP-NPs group. Even though H\&E staining of major organs showed negligible changes in histomorphology in all groups (Figure S11), serum biochemistry and hematological blood cell tests demonstrated up-regulated AST and ALT and decreased platelets and white blood cells respectively in CDDP and SFN+CDDP treated mice (Figure S12 and S13), indicating the potential hepatotoxicity and myelosuppression of CDDP. In comparison, SFN-CDDP-NPs had negligible effect on all tested biomarkers, which demonstrated its safe profile with reduced systemic toxicity of CDDP. 
To explore the potential of this nano-strategy, the therapeutic effect of SFN-CDDP-NPs was further investigated in a HepG2 liver cancer model with high-expression of GSH (Figure S14). ${ }^{44}$ In this study, two control groups, CDDP-loaded NPs without SFN (CDDP-NPs) (Figure S15) and CDDP-loaded NPs with free SFN (CDDP-NPs+SFN), were used to validate the nano-enabled co-delivery. As shown in Figure S16, SFN-CDDP-NPs treatment resulted in the most significant tumor growth inhibition with a TIR of $62.2 \%$, which was much higher than that of CDDP $(\mathrm{TIR}=19.1 \%), \mathrm{SFN}+\mathrm{CDDP}(\mathrm{TIR}=24.8 \%), \mathrm{CDDP}-\mathrm{NPs}(\mathrm{TIR}=31.7 \%)$ or CDDP-NPs+SFN (TIR = 39.1\%). Moreover, unlike CDDP or SFN+CDDP, SFN-CDDP-NPs treatment had no effect on mouse body weight (Figure S17). Mechanistic studies revealed that SFN-CDDP-NPs treatment led to the most significant cell apoptosis with the highest up-regulation of $\gamma$-H2AX, p53 and cleaved PARP and down-regulation of CDDP induced Bcl-2 expression (Figure S18). Collectively, these results strongly support that this nano-enable co-delivery strategy is applicable to treat tumor with high-expression of GSH.

\section{CONCLUSION}

In summary, SFN and CDDP co-loaded mPEG-PLGA NPs have been successfully prepared for enhanced breast cancer chemotherapy. After efficient internalization of SFN-CDDP-NPs by tumor cells, the more rapidly released SFN could notably decrease GSH content and thus significantly increase DNA-bound Pt, resulting in severe DNA damage and cellular apoptosis. Due to the improved chemosensitivity 
and preferential tumor accumulation, SFN-CDDP-NPs greatly inhibited orthotopic breast cancer progression with reduced toxic side effects. Moreover, the enhanced anti-tumor efficacy of SFN-CDDP-NPs was successfully extrapolated to a liver cancer model with high-expression of GSH. Taken together, NPs-enabled co-delivery of SFN and CDDP provides a simple but robust strategy to improve the sensitivity and therapeutic efficacy of Pt-based chemotherapy.

\section{EXPERIMENTAL SECTION}

Materials. Methoxy poly(ethylene glycol)-poly(lactide-co-glycolide) (mPEG-PLGA, molar ratio of D, L-lactic to glycolic acid, 75:25) was obtained from Jinan Daigang Biotechnology Co. Ltd (Jinan, China). Cisplatin (CDDP), was bought from Sigma-Aldrich. Sulforaphane (SFN) was obtained from Toronto Research Chemicals Inc. Poly ( $\gamma$, L-glutamic acid) ( $\gamma$-PGA, Mw 2000) was bought from Yuanye Bio-Technology Co., Ltd (Shanghai). LysoTracker Green DND 26 was obtained from Invitrogen. $35 \mathrm{~mm}$ confocal petri dishes (Cellvis, Mountain View, CA) were obtained from Hangzhou Xinyou Biotechnology Co., Ltd. (Hangzhou, China). RIPA lysis buffer (cat\# R0010) and Hoechst 33342 (cat\# C0030) were purchased from Solarbio Life Science (Beijing, China). Cell Counting Kit-8 (CCK-8) was bought from Dojindo Molecular Technologies (Tokyo, Japan).

Synthesis of $\gamma$-PGA-CDDP Conjugate. $\gamma$-PGA-CDDP conjugate was synthesized based on a reported method. ${ }^{33}$ Briefly, $20 \mathrm{mg}$ CDDP and $35 \mathrm{mg} \gamma$-PGA were 
dissolved in $5 \mathrm{ml}$ deionized water. The mixture was stirred for $48 \mathrm{~h}$ at $37^{\circ} \mathrm{C}$, followed by excessive dialysis against deionized water to remove free CDDP.

\section{Synthesis and Characterization of SFN-CDDP-NPs. SFN-CDDP-NPs was} fabricated using amphiphilic copolymer mPEG-PLGA by an adapted double emulsion method as previously described. ${ }^{38}$ Briefly, $1 \mathrm{~mL}$ of dichloromethane containing $10 \mathrm{mg}$ of mPEG-PLGA was mixed with $0.2 \mathrm{~mL}$ of $\gamma$-PGA-CDDP solution (0.5 mg CDDP) by sonication for $5 \mathrm{~min}$. The solution and $250 \mu \mathrm{g}$ SFN were added to $2 \mathrm{~mL}$ sodium cholate solution $(5 \%, \mathrm{w} / \mathrm{w})$ and further sonicated for $5 \mathrm{~min}$. The resulting emulsion was then added to $10 \mathrm{~mL}$ of sodium cholate solution $(1 \%$, w/w) drop-by-drop and further stirred for $10 \mathrm{~min}$. After removing dichloromethane through reduced vacuum evaporation, the resulting nanoparticles were obtained by centrifugation $(13,000 \mathrm{rpm}$, $10 \mathrm{~min}$ ) and washed with deionized water twice. The encapsulation efficiencies of CDDP and SFN were determined by inductively coupled plasma mass spectrometry (ICP-MS) and high-performance liquid chromatography (HPLC), respectively. By replacing $\gamma$-PGA-CDDP with fluorescent dyes, the RhoB-NPs and ICG-NPs were prepared similarly. After stained with $1 \%$ uranyl acetate, the nanoparticles were characterized by transmission electron microscopy (HT7700, HITACHI, Japan). The diameter, polydispersity index (PDI) and zeta potential of the nanoparticles were measured by DLS (Malvern Instruments Ltd., UK).

Drug Release in Vitro. SFN-CDDP-NPs were transferred into a dialysis bag (MWCO $1000 \mathrm{Da}$, Spectrum). The drug release was performed at $37{ }^{\circ} \mathrm{C}$ in $\mathrm{PBS}$ 
solution ( $\mathrm{pH} 7.4$ or $\mathrm{pH} 5.5)$. At the indicated time intervals, $0.2 \mathrm{~mL}$ of solution was acquired for SFN and CDDP quantification using HPLC and ICP-MS, respectively. $0.2 \mathrm{~mL}$ of fresh PBS was replenished immediately.

Cell Culture and Animals. The human breast cancer cell line MCF-7 and human hepatocellular carcinoma cell line HepG2 were cultured in DMEM (WISENT, Canada) containing $10 \%$ FBS (Gibco BRL, NY, USA) at $37{ }^{\circ} \mathrm{C}$ in a humidified atmosphere containing 5\% $\mathrm{CO}_{2}$. BALB/c nude mice (female, 6-8 weeks) were obtained from Vital River Laboratory Animal Technology Co. Ltd. The orthotropic breast tumor model was established by injecting $5 \times 10^{6} \mathrm{MCF}-7$ cells into the mammary fat pad of the mouse and the subcutaneous liver tumor model was generated by injecting $5 \times 10^{6}$ HepG2 cells into the right flank of the mouse. All animal experements were approved by the Institutional Animal Care and Use Committee of National Center for Nanoscience and Technology.

In Vitro Cytotoxicity Studies. MCF-7 cells were planted into 96-well plates (5,000 cells/well). $12 \mathrm{~h}$ later, the cells were incubated with SFN, CDDP, SFN+CDDP, SFN-CDDP-NP at different concentrations for another $24 \mathrm{~h}$. The cell viability was analyzed using a CCK-8 assay according to the manufacturer's protocols. The cytotoxicity of different formulations (10 $\mu \mathrm{M}$ SFN, $20 \mu \mathrm{M}$ CDDP) was also tested when cells were pre-treated with NAC $(1 \mathrm{mM})$ for $1 \mathrm{~h}$.

Cellular Uptake and Intracellular Localization. MCF-7 cells were planted in confocal dishes (Nunc, USA). After overnight incubation, cells were treated with free 
RhoB or RhoB-NPs for 1 or $6 \mathrm{~h}$, then washed for 3 times using PBS before imaging using a laser confocal fluorescence microscopy (LSM710, Carl Zeiss, Germany). The nucleus and lysosome were stained using Hochest 3342 and LysoTracker Green DND 26 , respectively.

For quantitatively comparing cellular uptake between free RhoB and RhoB-NPs, MCF-7 cells were planted in 12-well plates. When reaching 70\% confluent, cells were treated with free RhoB or RhoB-NPs for $6 \mathrm{~h}$. After being harvested and washed for 3 times using PBS, the cells were suspended in $0.2 \mathrm{ml}$ PBS buffer and analyzed using a Accuri C6 flow cytometer (BD, USA).

Cell Apoptosis Study. MCF-7 cells were planted into 12 -well plates $\left(1 \times 10^{4}\right.$ cells /well). After overnight incubation, cells were incubated with SFN, CDDP, SFN+CDDP and SFN-CDDP-NP for $24 \mathrm{~h}$. Cells were then harvested and co-stained with PI and Annexin V using an Annexin V-FITC Apoptosis Detection Kit (BD, USA). Finally, the apoptosis of cells was tested using flow cytometric analysis.

Western Blot Assay. MCF-7 cells were collected after different treatments and total cellular protein was extracted. The concentration of protein was measured by a bicinchoninic acid protein assay kit (cat\# 23225, Thermo). $50 \mu \mathrm{g}$ proteins were used for SDS-PAGE electrophoresis and further transferred to $0.22 \mu \mathrm{m}$ PVDF membranes. After incubated with p53 antibody (cat\# 10442-1-AP, Proteintech), Bcl-2 antibody (cat\# 12789-1-AP, Proteintech), PARP antibody (cat\# 13371-1-AP, Proteintech) and $\gamma-\mathrm{H} 2 \mathrm{AX}$ antibody (cat\# 39117, Active Motif) at $4{ }^{\circ} \mathrm{C}$ overnight, these membranes 
were washed for 3 times and incubated with secondary antibodies at RT for $1 \mathrm{~h}$. After washed for 3 times, the immuno-reactivity was visualized after incubating with enhanced chemiluminescence (ECL) reagents. GAPDH was chosen as a loading control.

In Vitro GSH Test. MCF-7 cells were planted in 6 well plate $\left(1 \times 10^{5}\right.$ cells/well $)$. After overnight incubation, cells were incubated with different formulations for another $6 \mathrm{~h}$. Then, a same number of cells was collected from each group, and cellular GSH content was quantified using a Reduced GSH Detection Kit (Solarbio, Beijing).

Quantification of DNA-bound Pt. The DNA platination was studied by quantification of DNA-bound Pt. Briefly, MCF-7 cells were planted into 6 well plate $\left(1 \times 10^{5}\right.$ cells/well $)$ for $12 \mathrm{~h}$. After treatment with different formulations for $6 \mathrm{~h}$, cells were collected and total DNA was extracted using a Qiagen QIAamp DNA Blood Mini Kit (Qiagen, Hangzhou). The extracted DNA were digested by aqua regia at $300^{\circ} \mathrm{C}$, and the concentration of DNA-bound Pt was determined by ICP-MS.

Pharmacokinetics and Bio-Distribution of NPs. Tumor-bearing mice were i.v. administrated with free ICG or ICG-NPs (ICG, $0.5 \mathrm{mg} / \mathrm{kg}$ ). At predetermined time intervals, blood was acquired and analyzed using the IVIS in vivo imaging system (Lumina XR Series III, PerkinElmer). To study the bio-distribution of free ICG and ICG-NPs, the treated tumor-bearing mice were imaged using the IVIS in vivo imaging system at predetermined time points. After 24 or $48 \mathrm{~h}$ post-treatment, mice were 
euthanized and major organs as well as tumor were acquired. The ex vivo fluorescent image was obtained using the IVIS in vivo imaging system.

Anti-Tumor Effects in Vivo. When tumor volume approached $150 \mathrm{~mm}^{3}$, mice were randomly divided to 6 groups $(n=5)$. Mice were i.v. administrated (3 times, every other day) with saline, SFN, CDDP, SFN+CDDP, SFN-CDDP-NPs or SFN-CDDP-NPs+NAC. The doses for SFN and CDDP were $0.59 \mathrm{mg} / \mathrm{kg}$ and $2 \mathrm{mg} / \mathrm{kg}$, respectively. NAC (10 $\mathrm{mg} / \mathrm{kg})$ was intraperitoneally administrated $1 \mathrm{~h}$ before SFN-CDDP-NPs treatment. The tumor volume and body weight of each mouse were recorded every other day. Tumor volume was calculated using the formula $\mathrm{V}=$ $0.5 \times \mathrm{L} \times \mathrm{W}^{2}$, where $\mathrm{L}$ represents the length and $\mathrm{W}$ represents the width. In the end, mice were euthanized, and the tumors were acquired and weighed.

To evaluate the anti-tumor activity in HepG2 tumor-bearing mice, mice were i.v. administrated (3 times, every other day) with saline, SFN, CDDP, SFN+CDDP, CDDP-NPs, CDDP-NPs+SFN, SFN-CDDP-NPs or SFN-CDDP-NPs+NAC.

Immunohistochemistry and TUNEL. The tumor was fixed with 4\% paraformaldehyde and embedded in paraffin, which was further cut into $5 \mu \mathrm{m}$ sections for immunohistochemical staining of p53, Bcl-2, $\gamma$-H2AX and cleaved PARP (cat\# 5625, Cell Signaling). To evaluate cellular apoptosis, the tumor section was stained using a terminal deoxynucleotidyl transferase-mediated deoxyuridine triphosphate nick end labeling (TUNEL) assay kit (KeyGEN BioTECH, Nanjing) according to the manufacturer's protocols. 
Hematology and Histopathology Examination. The murine blood was acquired from each group $(n=3)$ at the end of the experiment. After centrifugation at $4^{\circ} \mathrm{C}$ for 20 min, the plasma was collected for blood biochemical analysis. The concentrations of aspartate aminotransferase (AST), alkaline phosphatase (ALP) and alanine amino transferase (ALT) were determined to evaluate liver function. The concentrations of blood urea nitrogen (BUN) and creatinine (CRE) levels were determined to evaluate renal function. The whole blood was also collected for white blood cells, red blood cells and platelets analysis.

Hearts, livers, spleens, lungs and kidneys of the treated mice were harvested, fixed with 4\% paraformaldehyde and embedded in paraffin, which further stained with hematoxylin and eosin $(\mathrm{H} \& \mathrm{E})$ before undergoing histopathological examination using an optical microscope (AMG EVOS xl core, Life Technologies, USA).

Statistics. All experiments were conducted in triplicate unless elsewhere indicated. The result was presented as mean \pm standard deviation (S.D.). Student's $t$ test was used for statistical analysis. Difference between two groups was considered statistically significant when the two-sided $p<0.05$. 


\section{ASSOCIATED CONTENT}

The authors declare no competing financial interest.

\section{Supporting Information}

The Supporting Information is available free of charge on the ACS Publications website at DOI:

Additional experimental data such as DLS characterization, TEM characterization, Pt quantification, safety evaluation and HepG2 tumor model shown in Tables S1-S2 and Figures S1-S18 (PDF)

\section{AUTHOR INFORMATION}

\section{Corresponding Authors}

*E-mail: liyy@nanoctr.cn

*E-mail: y.bao@uea.ac.uk

*E-mail: niegj@nanoctr.cn

\section{Author Contributions}

${ }^{\#}$ Y.X. and X.H. contributed equally to this work.

\section{ACKNOWLEDGMENT}

This work was financially supported by National Key R\&D Program of China (Grant Nos. 2018YFA0208900, 2016YFA0201600), National Natural Science Foundation of China (Grant No. 31571021), Innovation Group of the National Natural Science Foundation of China (Grant No. 11621505), Frontier Research Program of the Chinese Academy of Sciences (Grant No. QYZDJ-SSW-SLH022), K.C.Wong Education Foundation (Grant No. GJTD-2018-03), the Key Laboratory of Biomedical Effects of Nanomaterials and Nanosafety, CAS, and an award from Cancer Prevention Research Trust, UK. 


\section{REFERENCES}

1. Siegel, R. L.; Miller, K. D.; Jemal, A. Cancer Statistics, 2019. Ca-Cancer J. Clin. 2019, 69, 7-34.

2. Silver, D. P.; Richardson, A. L.; Eklund, A. C.; Wang, Z. C.; Szallasi, Z.; Li, Q.; Juul, N.; Leong, C. O.; Calogrias, D.; Buraimoh, A.; Fatima, A.; Gelman, R. S.; Ryan, P. D.; Tung, N. M.; De Nicolo, A.; Ganesan, S.; Miron, A.; Colin, C.; Sgroi, D. C.; Ellisen, L. W.; et al. Efficacy of Neoadjuvant Cisplatin in Triple-Negative Breast Cancer. J. Clin. Oncol. 2010, 28, 1145-1153.

3. Guo, S.; Wang, Y.; Miao, L.; Xu, Z.; Lin, C. M.; Zhang, Y.; Huang, L. Lipid-Coated Cisplatin Nanoparticles Induce Neighboring Effect and Exhibit Enhanced Anticancer Efficacy. ACS Nano 2013, 7, 9896-9904.

4. Ling, X.; Chen, X.; Riddell, I. A.; Tao, W.; Wang, J. Q.; Hollett, G.; Lippard, S. J.; Farokhzad, O. C.; Shi, J. J.; Wu, J. Glutathione-Scavenging Poly(disulfide amide) Nanoparticles for the Effective Delivery of Pt(IV) Prodrugs and Reversal of Cisplatin Resistance. Nano Lett. 2018, 18, 4618-4625.

5. Kelland, L. The Resurgence of Platinum-Based Cancer Chemotherapy. Nat. Rev. Cancer 2007, 7, 573-584.

6. Yang, Y. T.; Shi, Y.; Jay, M.; Di Pasqua, A. J. Enhanced Toxicity of Cisplatin with Chemosensitizer Phenethyl Isothiocyanate toward Non-Small Cell Lung Cancer Cells When Delivered in Liposomal Nanoparticles. Chem. Res. Toxicol. 2014, 27, 946-948.

7. Uchino, H.; Matsumura, Y.; Negishi, T.; Koizumi, F.; Hayashi, T.; Honda, T.; Nishiyama, N.; Kataoka, K.; Naito, S.; Kakizoe, T. Cisplatin-Incorporating Polymeric Micelles (NC-6004) can Reduce Nephrotoxicity and Neurotoxicity of Cisplatin in Rats. Br. J. Cancer 2005, 93, 678-687.

8. Callari, M.; Aldrich-Wright, J. R.; de Souza, P. L.; Stenzel, M. H. Polymers with Platinum Drugs and Other Macromolecular Metal Complexes for Cancer Treatment. Prog. Polym. Sci. 2014, 39, 1614-1643. 
9. Li, Y.; Deng, Y.; Tian, X.; Ke, H.; Guo, M.; Zhu, A.; Yang, T.; Guo, Z.; Ge, Z.; Yang, X.; Chen, H. Multipronged Design of Light-Triggered Nanoparticles to Overcome Cisplatin Resistance for Efficient Ablation of Resistant Tumor. ACS Nano 2015, 9, 9626-9637.

10. Matsumura, Y.; Maeda, H. A New Concept for Macromolecular Therapeutics in Cancer Chemotherapy: Mechanism of Tumoritropic Accumulation of Proteins and the Antitumor Agent Smancs. Cancer Res. 1986, 46, 6387-6392.

11. Maeda, H. The Enhanced Permeability and Retention (EPR) Effect in Tumor Vasculature: The Key Role of Tumor-Selective Macromolecular Drug Targeting. $A d v$. Enzyme Regul. 2001, 41, 189-207.

12. Chen, H. B.; Gu, Z. J.; An, H. W.; Chen, C. Y.; Chen, J.; Cui, R.; Chen, S. Q.; Chen, W. H.; Chen, X. S.; Chen, X. Y.; Chen, Z.; Ding, B. Q.; Dong, Q.; Fan, Q.; Fu, T.; Hou, D. Y.; Jiang, Q.; Ke, H. T.; Jiang, X. Q.; Liu, G.; et al. Precise Nanomedicine for Intelligent Therapy of Cancer. Sci. China: Chem. 2018, 61, $1503-1552$.

13. Lee, C. M.; Tanaka, T.; Murai, T.; Kondo, M.; Kimura, J.; Su, W.; Kitagawa, T.; Ito, T.; Matsuda, H.; Miyasaka, M. Novel Chondroitin Sulfate-Binding Cationic Liposomes Loaded with Cisplatin Efficiently Suppress the Local Growth and Liver Metastasis of Tumor Cells In Vivo. Cancer Res. 2002, 62, 4282-4288.

14. Newman, M. S.; Colbern, G. T.; Working, P. K.; Engbers, C.; Amantea, M. A. Comparative Pharmacokinetics, Tissue Distribution, and Therapeutic Effectiveness of Cisplatin Encapsulated in Long-Circulating, Pegylated Liposomes (SPI-077) in Tumor-Bearing Mice. Cancer Chemother. Pharmacol. 1999, 43, 1-7.

15. Ye, H.; Jin, L.; Hu, R.; Yi, Z.; Li, J.; Wu, Y.; Xi, X.; Wu, Z. Poly(gamma,L-glutamic acid)-Cisplatin Conjugate Effectively Inhibits Human Breast Tumor Xenografted in Nude Mice. Biomaterials 2006, 27, 5958-5965.

16. Xiong, Y.; Jiang, W.; Shen, Y.; Li, H.; Sun, C.; Ouahab, A.; Tu, J. A Poly(gamma, L-glutamic acid)-Citric Acid Based Nanoconjugate for Cisplatin Delivery. Biomaterials 2012, 33, 7182-7193. 
17. Nishiyama, N.; Kato, Y.; Sugiyama, Y.; Kataoka, K. Cisplatin-Loaded Polymer-Metal Complex Micelle with Time-Modulated Decaying Property as A Novel Drug Delivery System. Pharm. Res. 2001, 18, 1035-1041.

18. Nishiyama, N.; Okazaki, S.; Cabral, H.; Miyamoto, M.; Kato, Y.; Sugiyama, Y.; Nishio, K.; Matsumura, Y.; Kataoka, K. Novel Cisplatin-Incorporated Polymeric Micelles can Eradicate Solid Tumors in Mice. Cancer Res. 2003, 63, 8977-8983.

19. Song, W. T.; Tang, Z. H.; Shen, N.; Yu, H. Y.; Jia, Y. J.; Zhang, D. W.; Jiang, J.; He, C. L.; Tian, H. Y.; Chen, X. S. Combining Disulfiram and Poly(L-glutamic acid)-Cisplatin Conjugates for Combating Cisplatin Resistance. J. Controlled Release 2016, 231, 94-102.

20. Plummer, R.; Wilson, R. H.; Calvert, H.; Boddy, A. V.; Griffin, M.; Sludden, J.; Tilby, M. J.; Eatock, M.; Pearson, D. G.; Ottley, C. J.; Matsumura, Y.; Kataoka, K.; Nishiya, T. A Phase I Clinical Study of Cisplatin-Incorporated Polymeric Micelles (NC-6004) in Patients with Solid Tumours. Br. J. Cancer 2011, 104, 593-598.

21. Stathopoulos, G. P.; Antoniou, D.; Dimitroulis, J.; Stathopoulos, J.; Marosis, K.; Michalopoulou, P. Comparison of Liposomal Cisplatin versus Cisplatin in Non-Squamous Cell Non-Small-Cell Lung Cancer. Cancer Chemother. Pharmacol. 2011, 68, 945-950.

22. Galluzzi, L.; Senovilla, L.; Vitale, I.; Michels, J.; Martins, I.; Kepp, O.; Castedo, M.; Kroemer, G. Molecular Mechanisms of Cisplatin Resistance. Oncogene 2012, 31, $1869-1883$.

23. Galluzzi, L.; Vitale, I.; Michels, J.; Brenner, C.; Szabadkai, G.; Harel-Bellan, A.; Castedo, M.; Kroemer, G. Systems Biology of Cisplatin Resistance: Past, Present and Future. Cell Death Dis. 2014, 5, e1257.

24. Kurokawa, H.; Ishida, T.; Nishio, K.; Arioka, H.; Sata, M.; Fukumoto, H.; Miura, M.; Saijo, N. Gamma-Glutamylcysteine Synthetase Gene Overexpression Results in Increased Activity of the ATP-Dependent Glutathione S-Conjugate Export Pump and Cisplatin Resistance. Biochem. Biophys. Res. Commun. 1995, 216, 258-264. 
25. Jakubikova, J.; Sedlak, J.; Mithen, R.; Bao, Y. Role of PI3K/Akt and MEK/ERK Signaling Pathways in Sulforaphane- and Erucin-Induced Phase II Enzymes and MRP2 Transcription, G2/M Arrest and Cell Death in Caco-2 Cells. Biochem. Pharmacol. 2005, 69, 1543-1552.

26. Clarke, J. D.; Dashwood, R. H.; Ho, E. Multi-Targeted Prevention of Cancer by Sulforaphane. Cancer Lett. 2008, 269, 291-304.

27. Harris, K. E.; Jeffery, E. H. Sulforaphane and Erucin Increase MRP1 and MRP2 in Human Carcinoma Cell Lines. J. Nutr. Biochem. 2008, 19, 246-254.

28. Mastrangelo, L.; Cassidy, A.; Mulholland, F.; Wang, W.; Bao, Y. Serotonin Receptors, Novel Targets of Sulforaphane Identified by Proteomic Analysis in Caco-2 Cells. Cancer Res. 2008, 68, 5487-5491.

29. Cheung, K. L.; Kong, A. N. Molecular Targets of Dietary Phenethyl Isothiocyanate and Sulforaphane for Cancer Chemoprevention. AAPS J. 2010, 12, 87-97.

30. Thornalley, P. J. Isothiocyanates: Mechanism of Cancer Chemopreventive Action. Anti-Cancer Drugs 2002, 13, 331-338.

31. Wang, W.; He, Y.; Yu, G.; Li, B.; Sexton, D. W.; Wileman, T.; Roberts, A. A.; Hamilton, C. J.; Liu, R.; Chao, Y.; Shan, Y.; Bao, Y. Sulforaphane Protects the Liver against CdSe Quantum Dot-Induced Cytotoxicity. PLoS One 2015, 10, e0138771.

32. Wang, H.; Zhao, Y.; Wu, Y.; Hu, Y. L.; Nan, K.; Nie, G.; Chen, H. Enhanced Anti-Tumor Efficacy by Co-Delivery of Doxorubicin and Paclitaxel with Amphiphilic Methoxy PEG-PLGA Copolymer Nanoparticles. Biomaterials 2011, 32, 8281-8290.

33. Gryparis, E. C.; Mattheolabakis, G.; Bikiaris, D.; Avgoustakis, K. Effect of Conditions of Preparation on the Size and Encapsulation Properties of PLGA-mPEG Nanoparticles of Cisplatin. Drug Delivery 2007, 14, 371-380.

34. Feng, Z.; Lai, Y.; Ye, H.; Huang, J.; Xi, X. G.; Wu, Z. Poly (gamma, L-glutamic acid)-Cisplatin Bioconjugate Exhibits Potent Antitumor Activity with Low Toxicity: A Comparative Study with Clinically Used Platinum Derivatives. Cancer Sci. 2010, $101,2476-2482$. 
35. Li, F.; Zhao, X.; Wang, H.; Zhao, R. F.; Ji, T. J.; Ren, H.; Anderson, G. J.; Nie, G. J.; Hao, J. H. Multiple Layer-by-Layer Lipid-Polymer Hybrid Nanoparticles for Improved FOLFIRINOX Chemotherapy in Pancreatic Tumor Models. Adv. Funct. Mater. 2015, 25, 788-798.

36. Cabral, H.; Matsumoto, Y.; Mizuno, K.; Chen, Q.; Murakami, M.; Kimura, M.; Terada, Y.; Kano, M. R.; Miyazono, K.; Uesaka, M.; Nishiyama, N.; Kataoka, K. Accumulation of Sub-100 nm Polymeric Micelles in Poorly Permeable Tumours Depends on Size. Nat. Nanotechnol. 2011, 6, 815-823.

37. Wang, H. X.; Zuo, Z. Q.; Du, J. Z.; Wang, Y. C.; Sun, R.; Cao, Z. T.; Ye, X. D.; Wang, J. L.; Leong, K. W.; Wang, J. Surface Charge Critically Affects Tumor Penetration and Therapeutic Efficacy of Cancer Nanomedicines. Nano Today 2016, $11,133-144$.

38. Zhao, X.; Yang, K. N.; Zhao, R. F.; Ji, T. J.; Wang, X. C.; Yang, X.; Zhang, Y. L.; Cheng, K. M.; Liu, S. L.; Hao, J. H.; Ren, H.; Leong, K. W.; Nie, G. J. Inducing Enhanced Immunogenic Cell Death with Nanocarrier-Based Drug Delivery Systems for Pancreatic Cancer Therapy. Biomaterials 2016, 102, 187-197.

39. Wang, H.; Wu, Y.; Zhao, R. F.; Nie, G. J. Engineering the Assemblies of Biomaterial Nanocarriers for Delivery of Multiple Theranostic Agents with Enhanced Antitumor Efficacy. Adv. Mater. 2013, 25, 1616-1622.

40. Carson, D. A.; Lois, A. Cancer Progression and P53. Lancet 1995, 346, 1009-1011.

41. Pledgie-Tracy, A.; Sobolewski, M. D.; Davidson, N. E. Sulforaphane Induces Cell Type-Specific Apoptosis in Human Breast Cancer Cell Lines. Mol. Cancer Ther. 2007, 6, 1013-1021.

42. Priya, D. K. D.; Gayathri, R.; Gunassekaran, G. R.; Murugan, S.; Sakthisekaran, D. Apoptotic Role of Natural Isothiocyanate from Broccoli (Brassica oleracea italica) in Experimental Chemical Lung Carcinogenesis. Pharm. Biol. 2013, 51, 621-628.

43. Ye, S.; Rao, J.; Qiu, S.; Zhao, J.; He, H.; Yan, Z.; Yang, T.; Deng, Y.; Ke, H.; Yang, H.; Zhao, Y.; Guo, Z.; Chen, H. Rational Design of Conjugated 
Photosensitizers with Controllable Photoconversion for Dually Cooperative Phototherapy. Adv. Mater. 2018, 30, 1801216.

44. Syng-Ai, C.; Kumari, A. L.; Khar, A. Effect of Curcumin on Normal and Tumor Cells: Role of Glutathione and Bcl-2. Mol. Cancer Ther. 2004, 3, 1101-1108. 
a

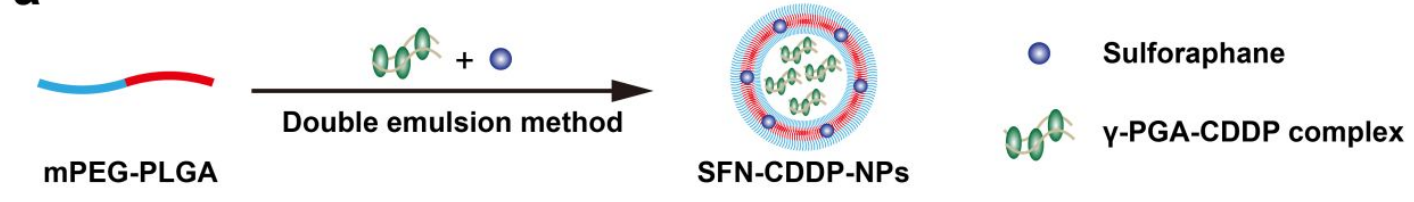

b

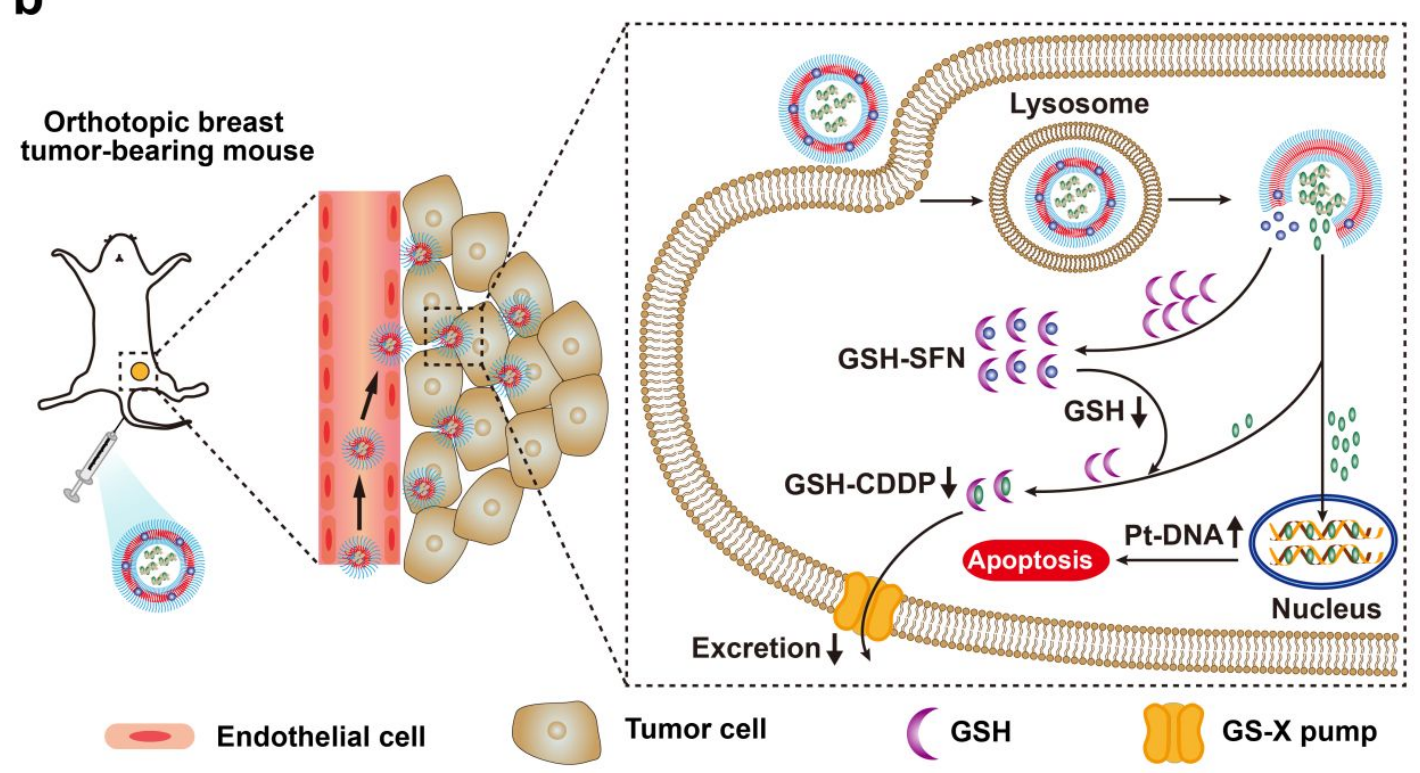

Scheme 1. Schematic diagram of the preparation of SFN-CDDP-NPs for improved anti-tumor therapy. (a) Schematic illustration of the synthesis of SFN-CDDP-NPs using a double emulsion method. (b) Proposed mechanism of SFN-CDDP-NPs for enhanced chemotherapy. The long circulating SFN-CDDP-NPs are accumulated in the orthotopic breast tumors via EPR effect. Endocytosis of SFN-CDDP-NPs allows the released SFN to deplete the abundant cellular GSH, which increases DNA platination-mediated cell apoptosis. 
a

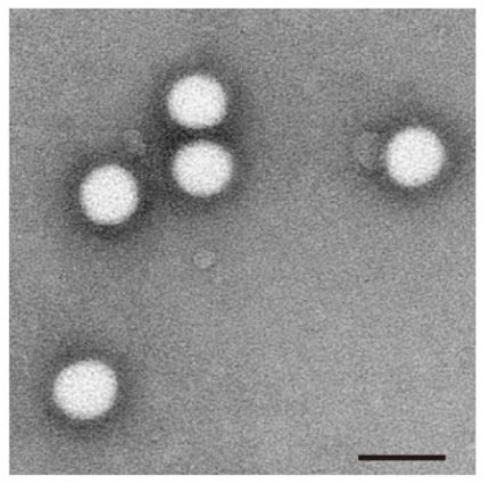

C

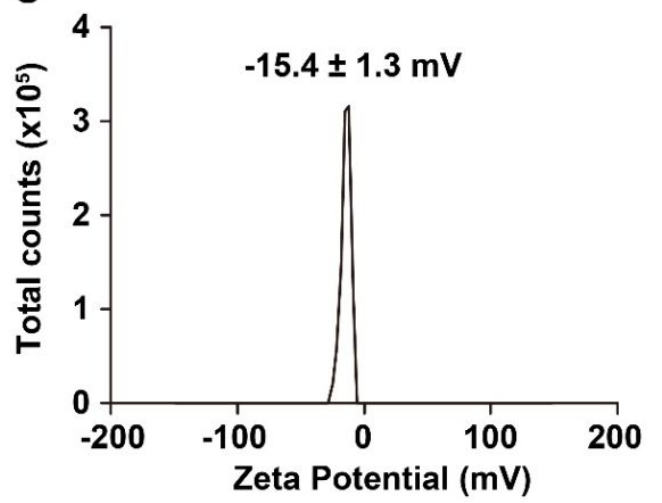

b

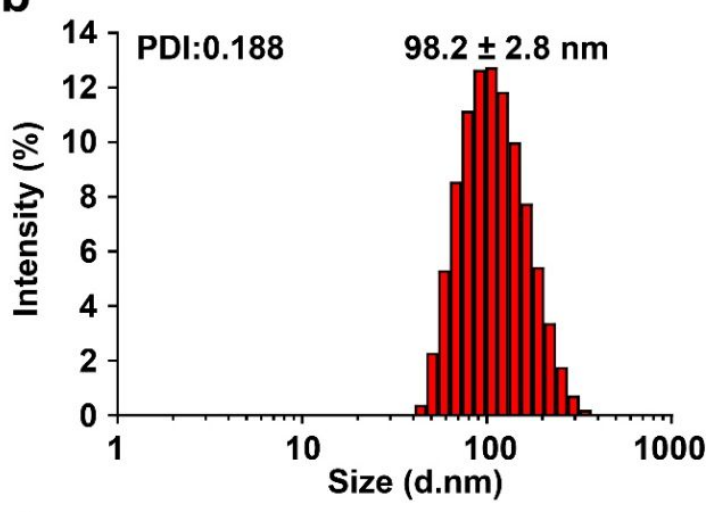

d

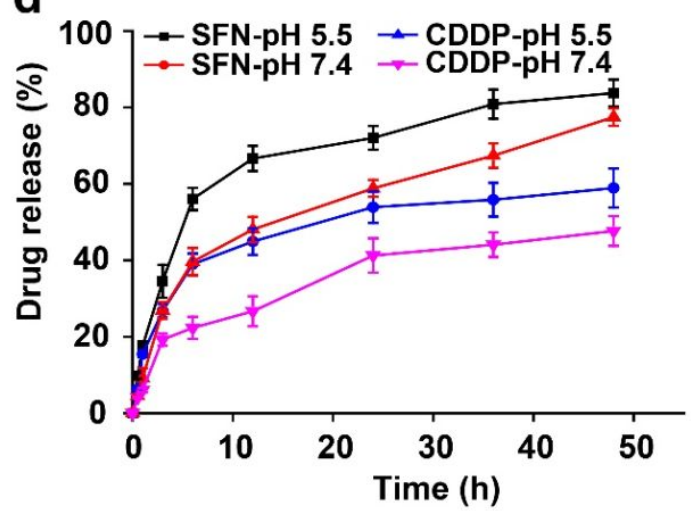

Figure 1. Characterization of SFN-CDDP-NPs. (a) TEM image of SFN-CDDP-NPs. Scale bar, $100 \mathrm{~nm}$. (b) Size distribution of SFN-CDDP-NPs. (c) Zeta potential of SFN-CDDP-NPs. (d) Release profiles of SFN and CDDP at pH 5.5 or 7.4. 
a
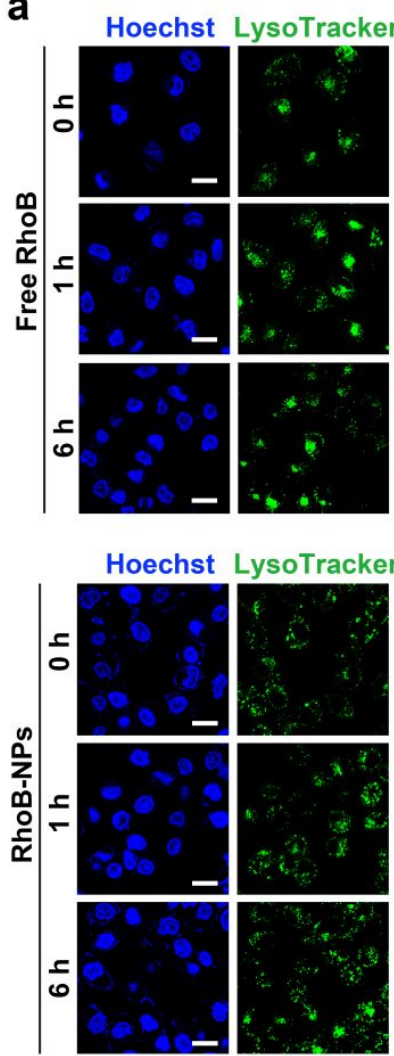

RhoB

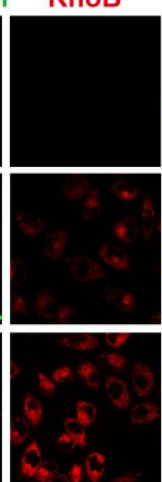

RhoB

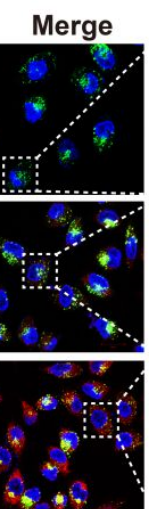

Merge
Enlarge

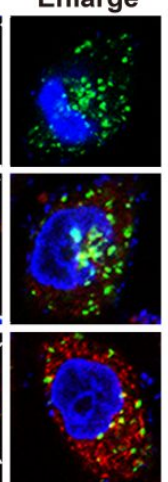

Enlarge
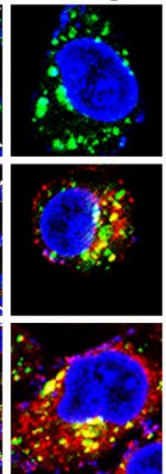

b

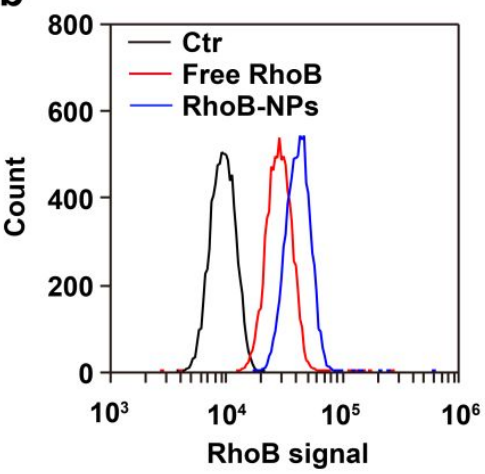

C

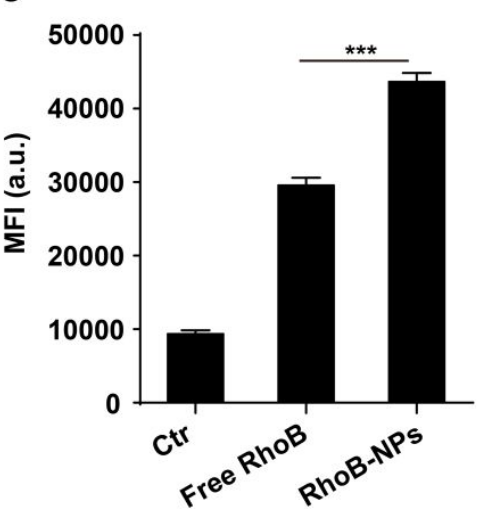

Figure 2. Cellular uptake and subcellular distribution of RhoB-NPs. (a) Confocal laser scanning microscope images of MCF-7 cells treated with RhoB or RhoB-NPs for different time. The nuclei were labeled with Hoechst 33342 (blue) and lysosomes were labeled with LysoTracker (green). Scale bars, $50 \mu \mathrm{m}$. (b) Flow cytometric analysis of RhoB signal in MCF-7 cells after incubated with free RhoB and RhoB-NPs for $6 \mathrm{~h}$. (c) Quantification of RhoB signal in MCF-7 cells. Data are presented as means $\pm \mathrm{SD}(\mathrm{n}=3) .{ }^{* * *} P<0.001$. 
a

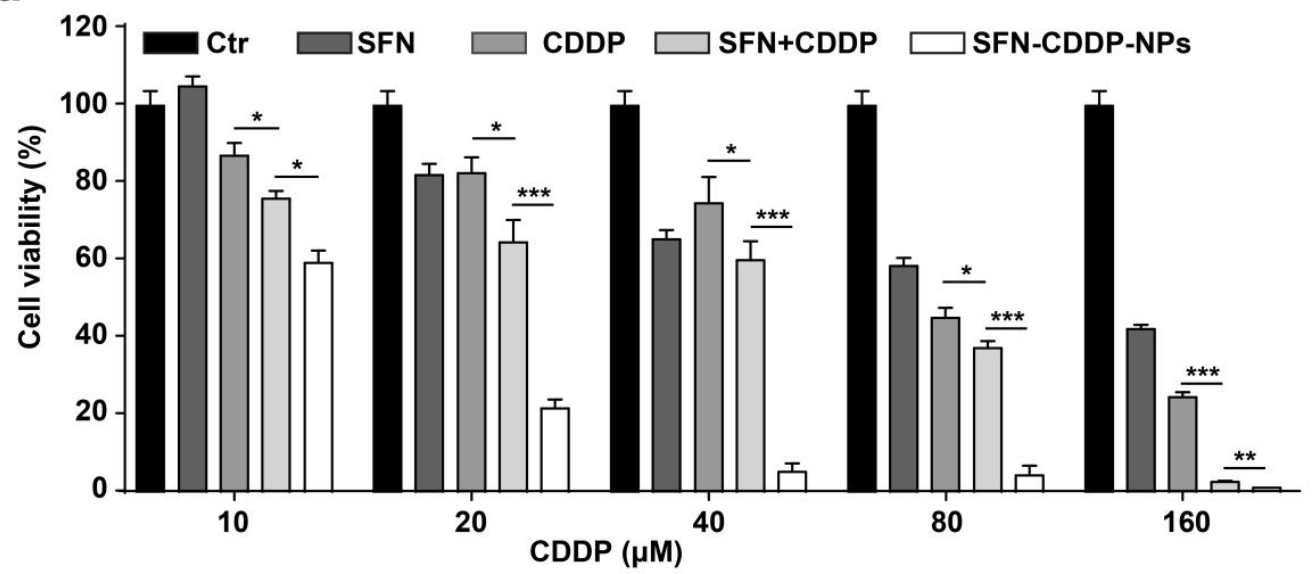

b
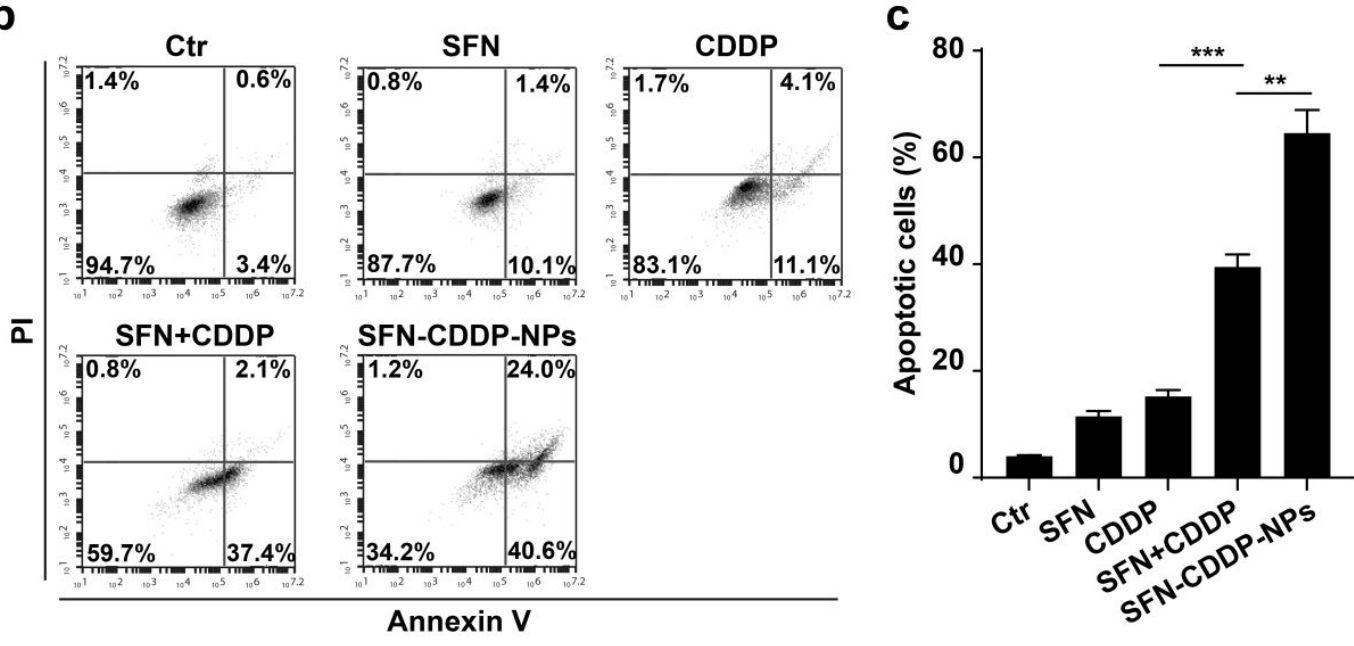

Figure 3. Evaluation of cytotoxicity. (a) Cytotoxicity of MCF-7 cells after different treatments for 24 h. (b) Apoptosis study of MCF-7 cells after different treatments for 24 h. (c) Quantitative analysis of apoptotic cells. Data are presented as means \pm SD (n 3). ${ }^{*} P<0.05, * * P<0.01, * * * P<0.001$. 
a

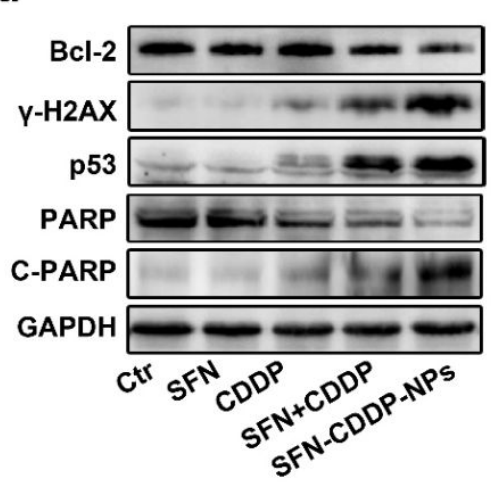

C

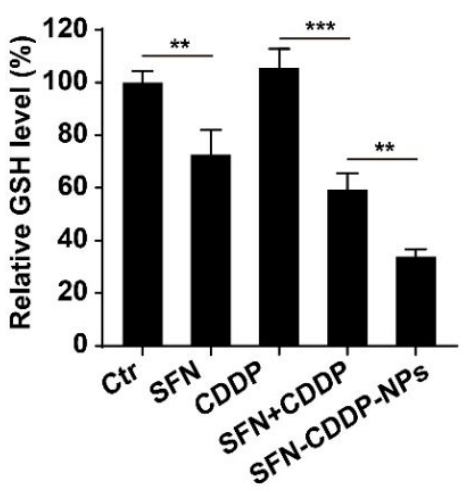

b

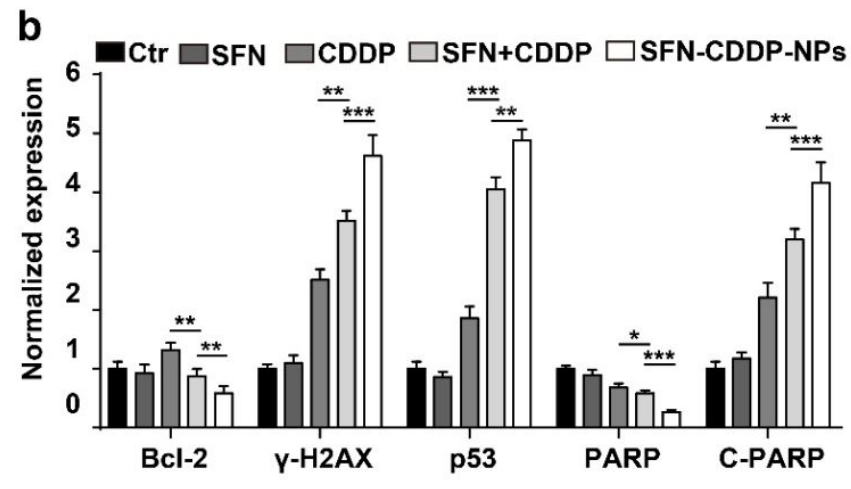

d

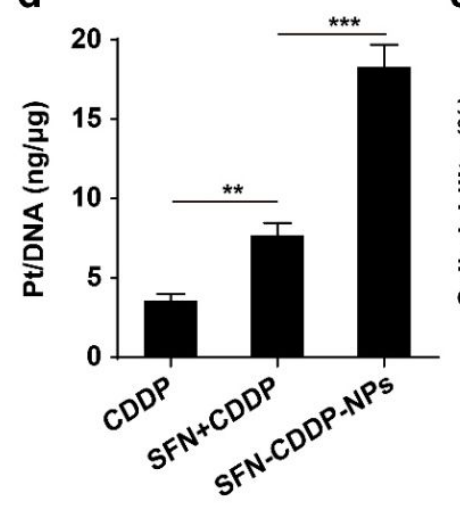

e

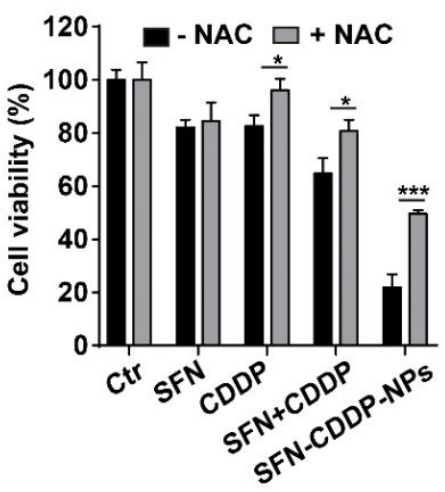

Figure 4. Mechanism analysis of SFN-CDDP-NPs induced cell apoptosis. (a)

Western blot analysis after different treatments for $6 \mathrm{~h}$. (b) Statistical analysis of the protein expression normalized to control. (c) Quantification of cellular GSH after different treatments for $6 \mathrm{~h}$. (d) Pt content in the genomic DNA of MCF-7 cells after different treatments for $6 \mathrm{~h}$. (e) Cell viability after different treatments for $24 \mathrm{~h}$ with or without NAC pretreatment. Data are presented as means $\pm \mathrm{SD}(\mathrm{n}=3) .{ }^{*} P<0.05,{ }^{*} P$ $<0.01, * * * P<0.001$. 


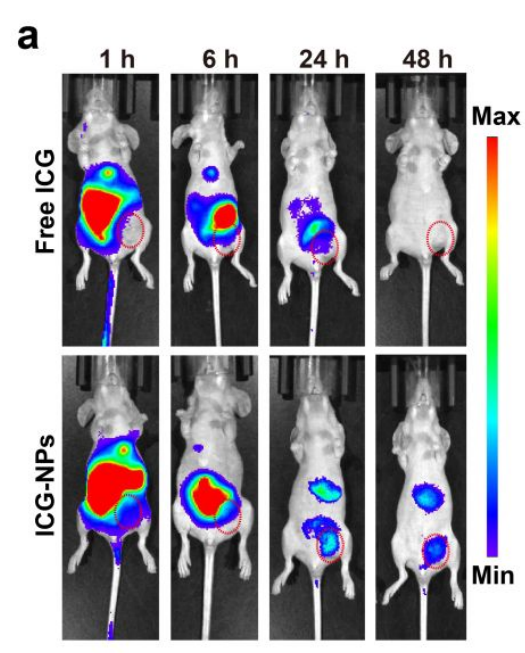

C

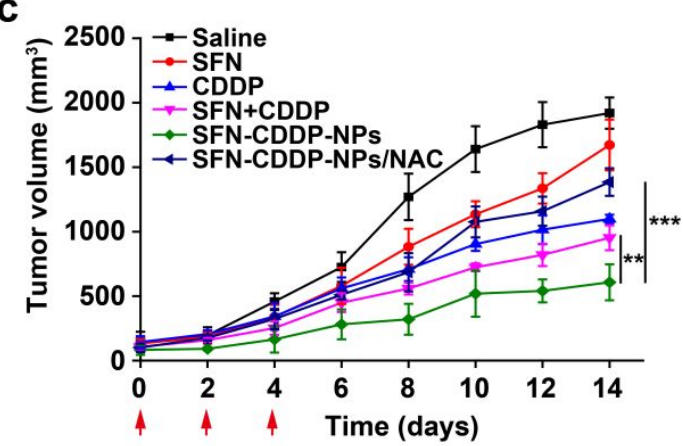

e

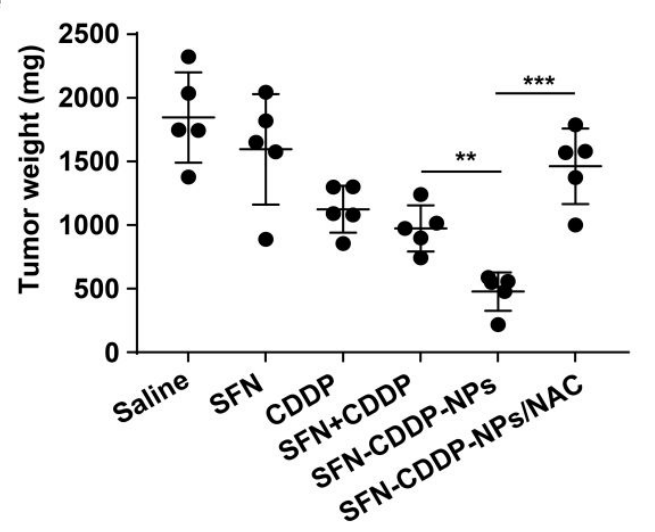

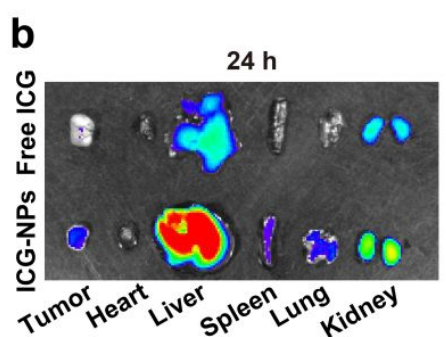
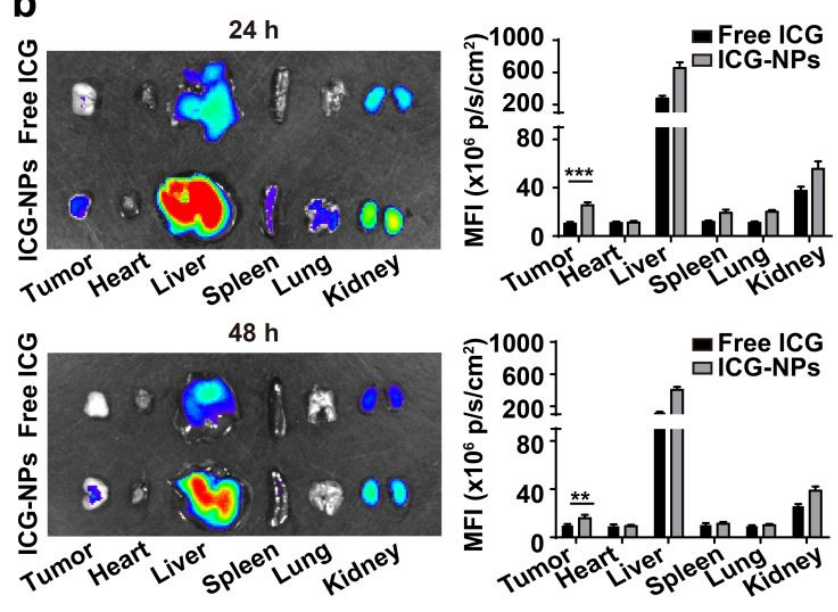

d

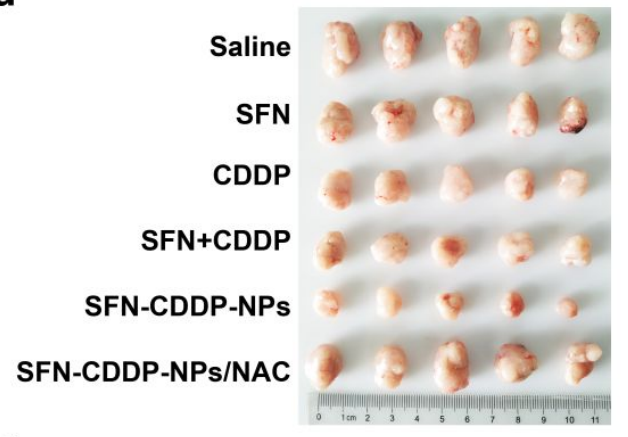

f

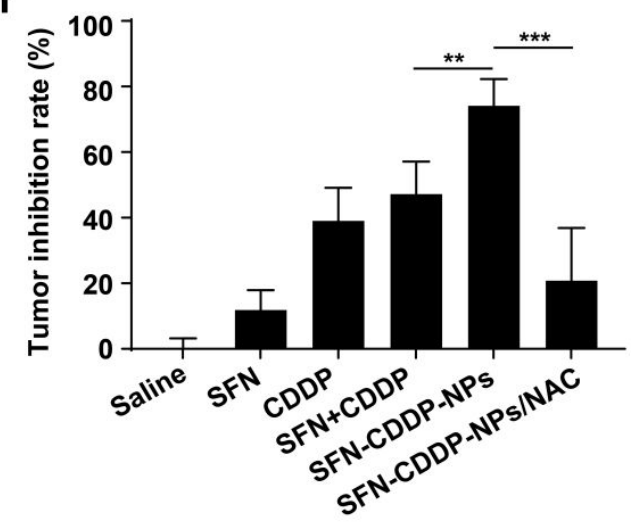

Figure 5. Bio-distribution and antitumor activity of SFN-CDDP-NPs in vivo. (a)

In vivo fluorescence images of MCF-7 tumor-bearing mice at different time points. (b) Ex vivo fluorescence images of major organs as well as tumors, and their corresponding mean fluorescence intensity (MFI) analysis at $24 \mathrm{~h}$ and $48 \mathrm{~h}$ post-injection. (c) Tumor growth curves. (d) Photos of tumors from different groups. (e) Tumor weights at the end of experiment. (f) Tumor inhibition rates of different groups. Data are presented as means $\pm \mathrm{SD}(\mathrm{n}=5)$. $* * P<0.01, * * * P<0.001$. 


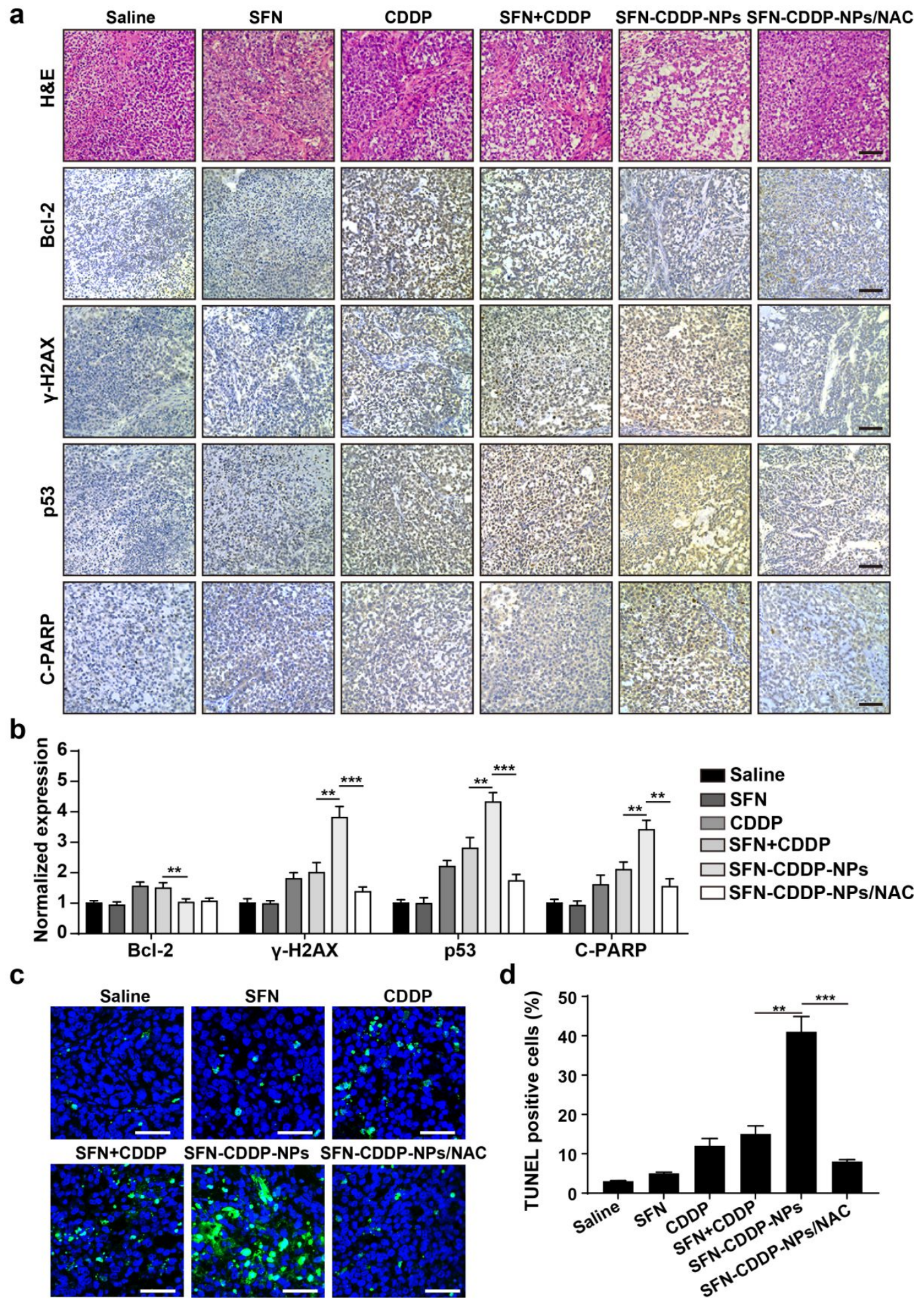

Figure 6. Apoptotic analysis of MCF-7 tumors. (a) H\&E and IHC images of tumor sections. Scale bars, $200 \mu \mathrm{m}$. (b) Statistical analysis of protein expression normalized to saline group. (c) TUNEL analysis of tumor section. Scale bars, $100 \mu \mathrm{m}$. (d) Quantification of TUNEL-positive cells. Data are presented as means $\pm \operatorname{SD}(n=5)$. $* * P<0.01, * * * P<0.001$. 


\section{Table of contents (TOC) graphic}

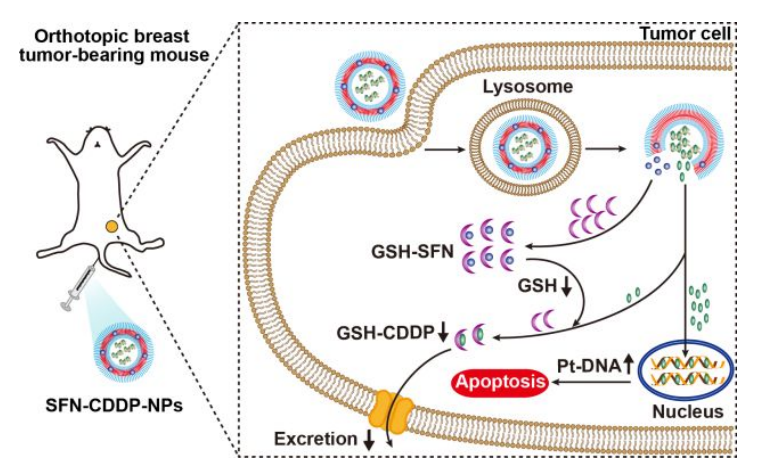

Polymeric nanoparticles are utilized to co-deliver a water-soluble cisplatin (CDDP) derivative, poly ( $\gamma$, L-glutamic acid)-CDDP conjugate, and sulforaphane, a naturally occurring compound derived from broccoli, that can achieve efficient glutathione (GSH) depletion, to increase the chemosensitivity of CDDP. Endocytosis of SFN-CDDP-NPs allows the released SFN to deplete the abundant cellular GSH, which increases DNA platination-mediated cell apoptosis. 